\title{
Different Cosmetic Habits Can Affect the Biophysical Profile of Facial Skin: A Study of Korean and Chinese Women
}

\author{
Ji Su Lee, Jaehyoun $\mathrm{Ha}^{1}$, Kyeho Shin ${ }^{2}$, Hyojung Kim ${ }^{3}$, Soyun Cho \\ Department of Dermatology, SMG-SNU Boramae Medical Center, Seoul, ${ }^{1}$ Skin Research Institute, IEC Korea, ${ }^{2}$ Department of Beauty \\ Coordination, Suwon Science College, Suwon, ${ }^{3}$ Smart Beauty Life Lab, SKIN79, Inc., Seoul, Korea
}

Background: Previous studies on the age-, climate, and skin care habit-related changes of biophysical parameters have mainly focused on Caucasians, and studies on Asians are in paucity. Objective: This study was aimed to investigate the variations of cutaneous biophysical parameters in Chinese and Korean women (northeast Asians) and to assess the association between those parameters and age, climate, and cosmetic habits. Methods: A cross-sectional study included 361 healthy Chinese and Korean women between 18 and 49 years of age in 4 cities (Guangzhou, Nanjing, and Shijiazhuang in China, and Suwon in Korea). We measured skin surface temperature, hydration, transepidermal water loss (TEWL), sebum, elasticity, skin pore, wrinkle, and skin tone (brightness) using non-invasive instruments. Demographic profiles and cosmetic habits were assessed using a questionnaire. Results: Skin elasticity and tone decreased, and pore size and wrinkle increased with age. Subjects in Suwon (Korean) showed higher hydration level, lower TEWL and lower sebum, less severe wrinkle and brighter skin than those in the 3 cities in China. After adjusting for age and region, using sunscreen everyday, wearing base makeup daily, and using moisturizers improved hydration, TEWL, and

Received July 18, 2018, Revised October 23, 2018, Accepted for publication October 25, 2018

Corresponding author: Soyun Cho, Department of Dermatology, SMG-SNU Boramae Medical Center, 20 Boramae-ro 5-gil, Dongjak-gu, Seoul 07061, Korea. Tel: 82-2-870-2381, Fax: 82-2-870-3866, E-mail: sycho@snu.ac.kr ORCID: https://orcid.org/0000-0003-2468-485X

This is an Open Access article distributed under the terms of the Creative Commons Attribution Non-Commercial License (http://creativecommons. org/licenses/by-nc/4.0) which permits unrestricted non-commercial use, distribution, and reproduction in any medium, provided the original work is properly cited.

Copyright (c) The Korean Dermatological Association and The Korean Society for Investigative Dermatology elasticity significantly. Conclusion: Women in Suwon (Korea) were found to have a better profile of biophysical parameters than women in the 3 Chinese cities, which might be attributed to cosmetic habits, besides age and climatic factors. The fact that appropriate cosmetic habits are associated with favorable skin biophysical parameters underscores the importance of daily skin care routine in preserving skin functions. (Ann Dermatol 31(2) $175 \sim 185,2019$ )

\section{-Keywords-}

Aging, Asian, Biophysical parameters, Climate, Cosmetics

\section{INTRODUCTION}

Skin is the largest and outermost human organ, and plays various important functions, such as forming a protective barrier to exogenous factors, modulating the passage of the water and electrolytes, and thermoregulation ${ }^{1-3}$. Various internal and external factors can affect functional state of skin, and biophysical parameters which are measured using commercial non-invasive methods are known to reflect the changes of skin conditions ${ }^{2,4}$. Understanding the changes in biophysical characteristics of the skin may be useful for developing a proper approach to skin care and to predict and manage skin diseases ${ }^{3,5,6}$. Although there are a few previous studies on the age- ${ }^{4-15}$, climate- ${ }^{1,16}$, and skin care habit-related ${ }^{2,8}$ changes in biophysical parameters of Caucasian women, there are a limited number of reports for Asian women. This study aimed to investigate the variations of biophysical parameters in Chinese and Korean women (northeast Asians) and to assess the association between these parameters and age, climate, and cosmetic habits. 


\section{MATERIALS AND METHODS}

\section{Study subjects}

As representatives of northeast Asia, 3 east cities (Guangzhou, Nanjing, and Shijiazhuang) in China and Suwon in Republic of Korea were selected (Fig. 1). Guangzhou and Nanjing were selected as warm cities, and Shijiazhuang and Suwon as cold cities. In each temperature group, Nanjing and Suwon were selected as humid cities, Guangzhou and Shijiazhuang as dry cities. The data were obtained between December 21, 2015 and January 15, 2016. Geographic data of the 4 cities and climatic information during the study period are summarized in Table 1. The study aimed to recruit more than 80 healthy women from each city. In each city, at least 20 women were recruited by each age group: 18 21 years, $22 \sim 25$ years, 26 37 years, and 38 49 years. Since the study was designed for the purpose of developing skin care products for certain social stages of life, age group was divided into college years, early employment years, mid-twenties to late-thirties, and late-thirties to just before 50 years of age. All subjects had lived in each city for at least 5 years. Subjects who were pregnant, lactating, or planning a pregnancy were excluded. None of the subjects had significant skin disorder, had taken any medications that could interfere with the study results, or had undergone any skin treatments (i.e., chemical peel, or laser treatments) within 6 months prior to the study. Finally, a total of 361 healthy Chinese and Korean women between 18 and 49 years of age in 4 cities were enrolled. This study was approved by the Institutional Review Board at IEC Korea (No. IECK (1)-IRB-15-K254443) and was conducted with strict adherence to the Declaration of Helsinki Principles.

\section{Study protocol}

Guangzhou and Nanjing (grouped as warm cities) showed higher mean temperature than Shijiazhuang and Suwon (grouped as cold cities) $(p=0.000)$. In each group, Nanjing

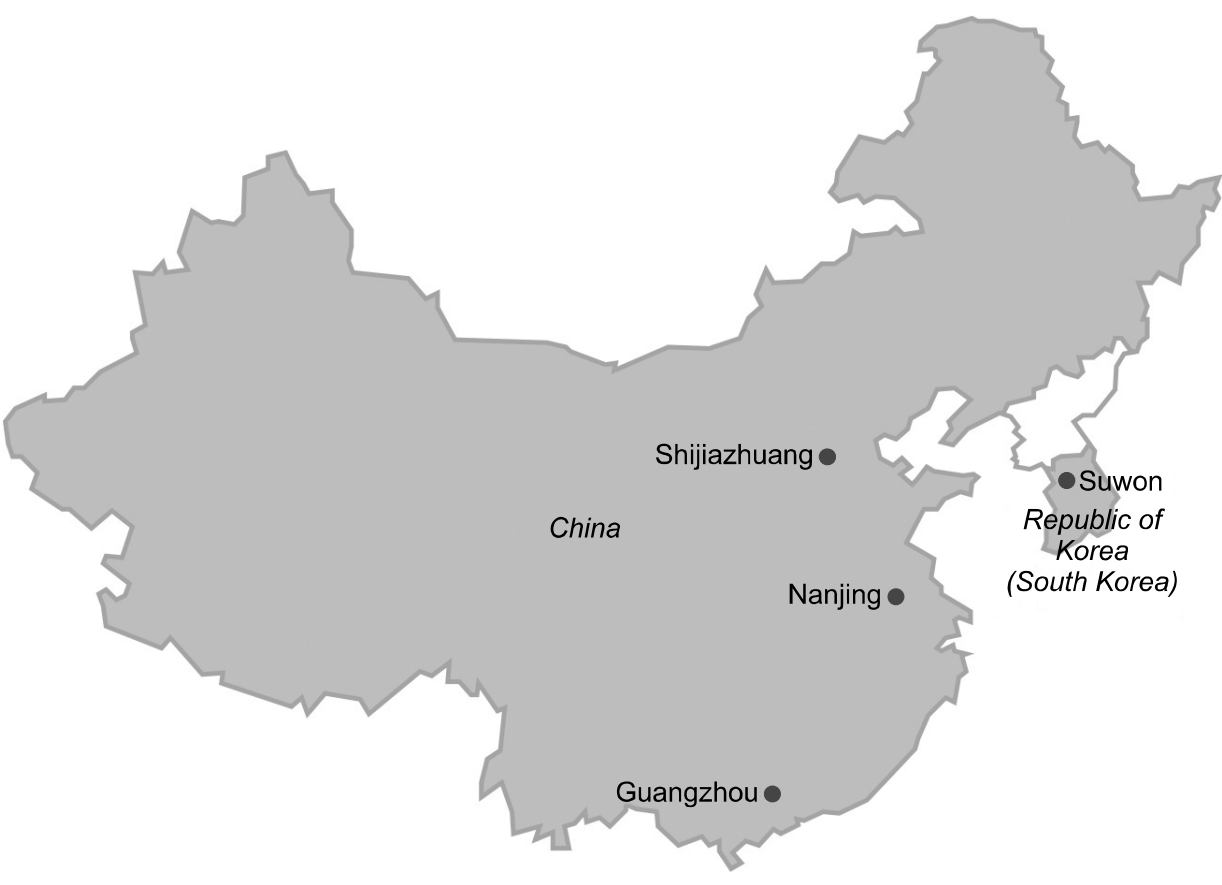

Fig. 1. The location of 4 cities on the map.

Table 1. Geographic information of 4 cities and climatic information during the study period*

\begin{tabular}{lllllll}
\hline \multicolumn{1}{c}{ City (country) } & \multicolumn{1}{c}{ Longitude } & \multicolumn{1}{c}{ Latitude } & Mean temperature $\left({ }^{\circ} \mathrm{C}\right)^{\dagger}$ & Relative humidity $(\%)$ & Wind speed $(\mathrm{km} / \mathrm{h})^{\ddagger}$ \\
\hline Guangzhou (China) & $113^{\circ} 15^{\prime} 0^{\prime \prime} \mathrm{E}$ & $23^{\circ} 7^{\prime} 0.01 " \mathrm{~N}$ & $16.7 \pm 2.8(10,25)$ & $79.6 \pm 9.7(38,100)$ & $8.4 \pm 4.6(2.3,22.5)$ \\
Nanjing (China) & $118^{\circ} 46^{\prime} 40.01^{\prime \prime} \mathrm{E}$ & $32^{\circ} 3^{\prime} 42.01 " \mathrm{~N}$ & $16.0 \pm 2.0(11,23)$ & $81.5 \pm 13.5(31,100)$ & $6.3 \pm 3.3(2.3,16.8)$ \\
Shijiazhuang (China) & $114^{\circ} 28^{\prime} 43^{\prime \prime} \mathrm{E}$ & $38^{\circ} 2^{\prime} 29^{\prime \prime} \mathrm{N}$ & $-1.8 \pm 1.8(-11,10)$ & $70.2 \pm 17.6(8,100)$ & $3.1 \pm 1.3(1.8,6.0)$ \\
Suwon (Republic of Korea) & $127^{\circ} 0^{\prime} 32^{\prime \prime} \mathrm{E}$ & $37^{\circ} 17^{\prime} 28 \mathrm{~N}$ & $-0.5 \pm 3.4(-11,11)$ & $74.1 \pm 12.9(24,100)$ & $6.5 \pm 1.7(2.8,10.8)$ \\
\hline
\end{tabular}

Values are presented as mean \pm standard deviation (minimum, maximum). *Data were obtained from following websites: http:// latitudelongitude.org, https://www.timeanddate.com. ${ }^{\dagger} \mathrm{A}$ mean value of the mean of daily temperature. ${ }^{\ddagger} \mathrm{A}$ mean value of the simple scalar mean of daily wind speed. 
was more humid than Guangzhou $(p=0.000)$, and Suwon was more humid than Shijiazhuang $(p=0.000)$.

On the visit day, volunteers were required not to apply any cosmetics on their face. Once volunteers visited, skin surface temperature was taken from the cheek and forehead without acclimatization. Then they were instructed to read the research protocol that explained the study procedure before giving informed consent. They were required to complete a questionnaire to assess demographic profiles and use of cosmetics. Then, they washed their face with warm water without any cleansers or soap, and measurements were performed by multiple non-invasive devices for the following parameters: hydration, transepidermal water loss (TEWL), sebum, elasticity, skin pore, wrinkle, and skin tone (brightness). All study protocols were implemented in an environment-controlled room (temperature, $22^{\circ} \mathrm{C} \pm 2^{\circ} \mathrm{C}$ and relative humidity, $50 \% \pm 10 \%$ ).

\section{Measurements}

Skin surface temperature was measured using a non-contact infrared thermometer. Skin electrical capacitance was measured using a Corneometer CM825 (Courage + Khazaka Electronic, Cologne, Germany), shown in system-specific arbitrary units (AU). This parameter was used to estimate the hydration level of stratum corneum. Higher values mean higher hydration level. TEWL was measured using Vapometer (Delfin Technologies, Kuopio, Finland), expressed in $\mathrm{g} / \mathrm{m}^{2}$. Higher values mean more water loss through the epidermis. Measurements of hydration and TEWL were performed 3 times from the cheek and forehead, and the average was calculated for each region. Sebum output was measured using a Sebumeter SM810 (Courage+Khazaka Electronic $\mathrm{GmbH}$ ), expressed in $\mu \mathrm{g} / \mathrm{cm}^{2}$. Higher values mean more sebum secretion. The collection was performed once, each from the cheek and forehead. Skin elasticity of the cheek was measured by Cutometer MPA580 (Courage + Khazaka Electronic $\mathrm{GmbH}$ ), a suction chamber device. R2, Ua/Uf, is considered as the main parameter that represents the skin's ability to regain its initial position after deformation and was most often used in previous studies ${ }^{1}$. Values close to 1 mean high elasticity. Skin pore area, wrinkle, and skin tone (brightness) were measured using Janus (PSI, Seoul, Korea), expressed in a 100-percentage scale ${ }^{17-20}$. Photographs of faces were taken at 3 different angles (frontal, left lateral, and right lateral) with a 10 megapixel ultra-high resolution camera, and were analyzed using built-in image analysis software. The extent of pore widening was assessed from forehead, nose, and both cheeks, and the degree of skin wrinkles was assessed from the crow's feet. The average of red, green, and blue values of the cheeks, nose, eyes, and fore- head was calculated and converted to a 100-point skin tone grading. Higher values mean more pore widening, severe wrinkles, and brighter skin.

\section{1) Statistical analysis}

Temperature and humidity comparison between 4 cities were made using the generalized estimating equation. Simple linear regression was performed to assess the association between biophysical parameters and age. Group comparison was made using the one-way (analysis of variance) ANOVA and post-hoc pairwise comparison (Scheffe method) was performed in each group comparison for continuous variables (biophysical parameters). For ordinal variables (cosmetic habit items), Mann-Whitney test was used for group comparison. Age- and region-adjusted multiple linear regression was performed to assess the association between biophysical parameters and cosmetic habits. $p$-value less than 0.05 was considered statistically significant. Data were summarized using mean (standard deviation) for continuous variables and frequency (percentage) for numerical variables. Statistical tests were performed using IBM SPSS Statistics ver. 19.0 (IBM Co., Armonk, NY, USA).

\section{RESULTS}

\section{Biophysical parameters according to age}

Variations of biophysical parameters according to age are shown in Fig. 2 and Supplementary Table 1. Skin surface temperature, hydration, TEWL, and sebum excretion showed values of determination coefficient ( $R$-squared, $R^{2}$ ) less than 0.1 (Fig. 2A D ). Skin elasticity and tone decreased, and skin pore and wrinkle increased with age (Fig. $2 \mathrm{E} \sim \mathrm{H}$ ); among the 4 parameters, wrinkle showed the largest determination coefficient $\left(\mathrm{R}^{2}=0.455\right)$ (Fig. $2 \mathrm{~F} \sim \mathrm{H}$ ).

\section{Biophysical parameters according to geographical region}

Variations of biophysical parameters according to region are shown in Fig. 3 and Table 2. Skin surface temperature of the cheek and TEWL were lower in Suwon than the 3 cities in China (Fig. 3A, C). Nanjing and Suwon, which are more humid in each temperature group, showed much higher hydration level (Fig. 3B).

Sebum output was higher in warm cities than cold cities (Fig. 3D). Skin elasticity and pore size were not significantly different among 4 cities (Fig. 3E, F). The severity of wrinkle was the mildest and skin tone the brightest in Suwon among the 4 cities (Fig. 3G, H).

\section{Cosmetic habits according to country}

All assessed cosmetic habits were significantly different 
A
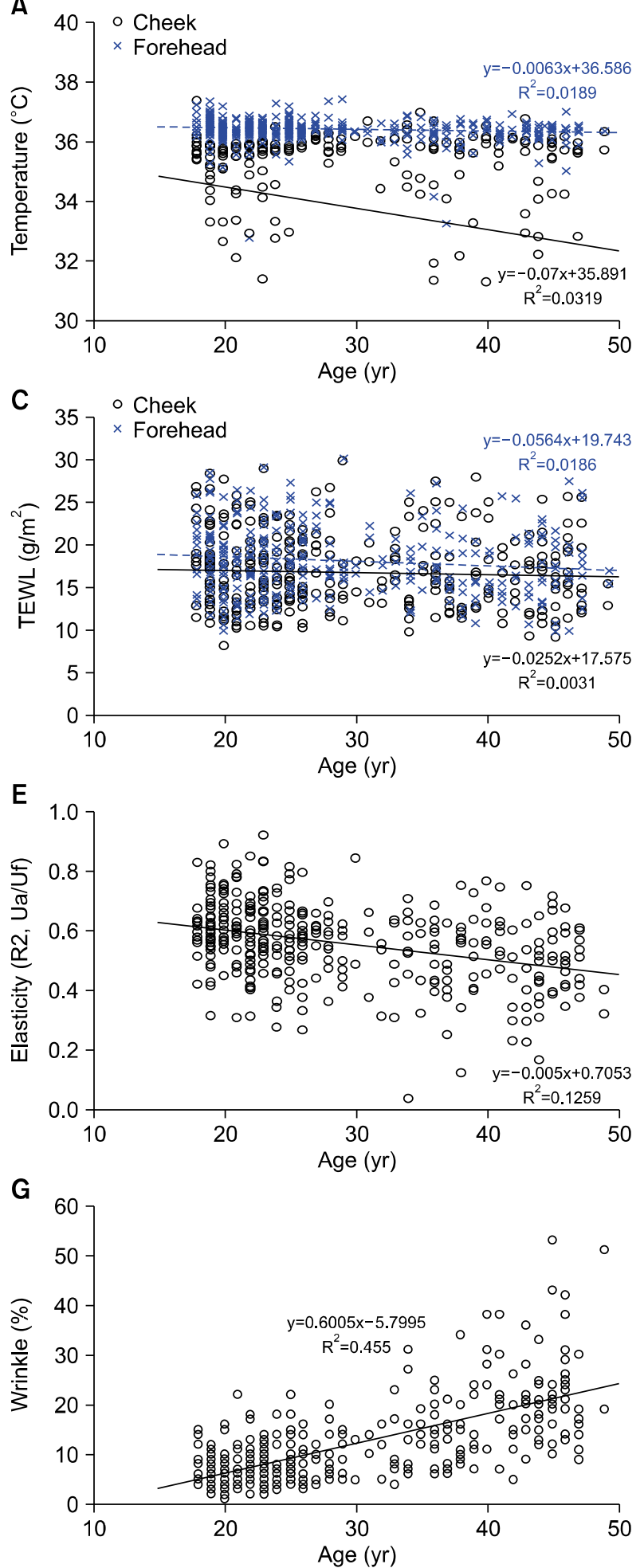

B
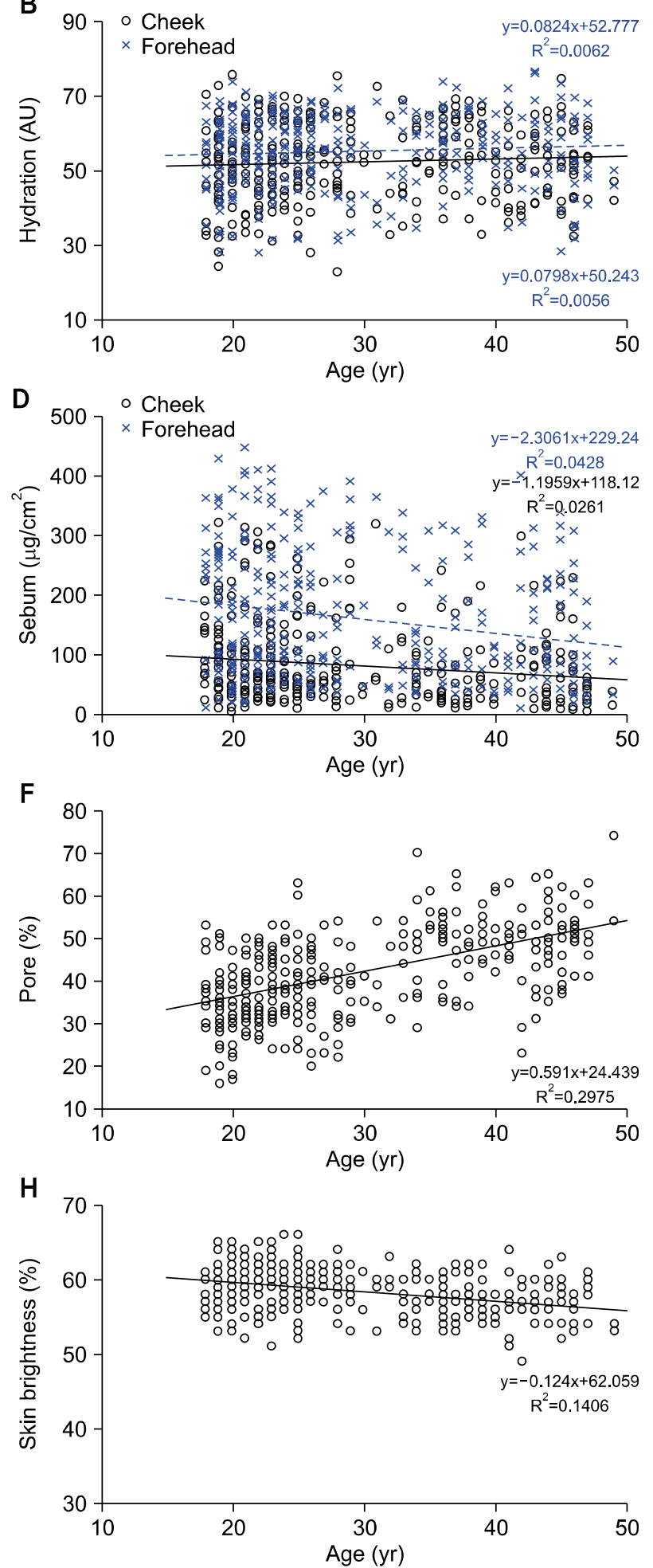

Fig. 2. Variation of biophysical parameters according to age. (A $\sim \mathrm{D})$ Skin surface temperature, hydration level, transepidermal water loss (TEWL), and sebum excretion showed very weak strength of determination coefficient (R-squared, $R^{2}$ ). (E, $F$, and $H$ ) Skin elasticity and skin tone decreased and skin pore increased with age, and they showed weak strength of determination coefficient. (G) Wrinkle incresed with age and showed moderate strength of determination coefficient. 

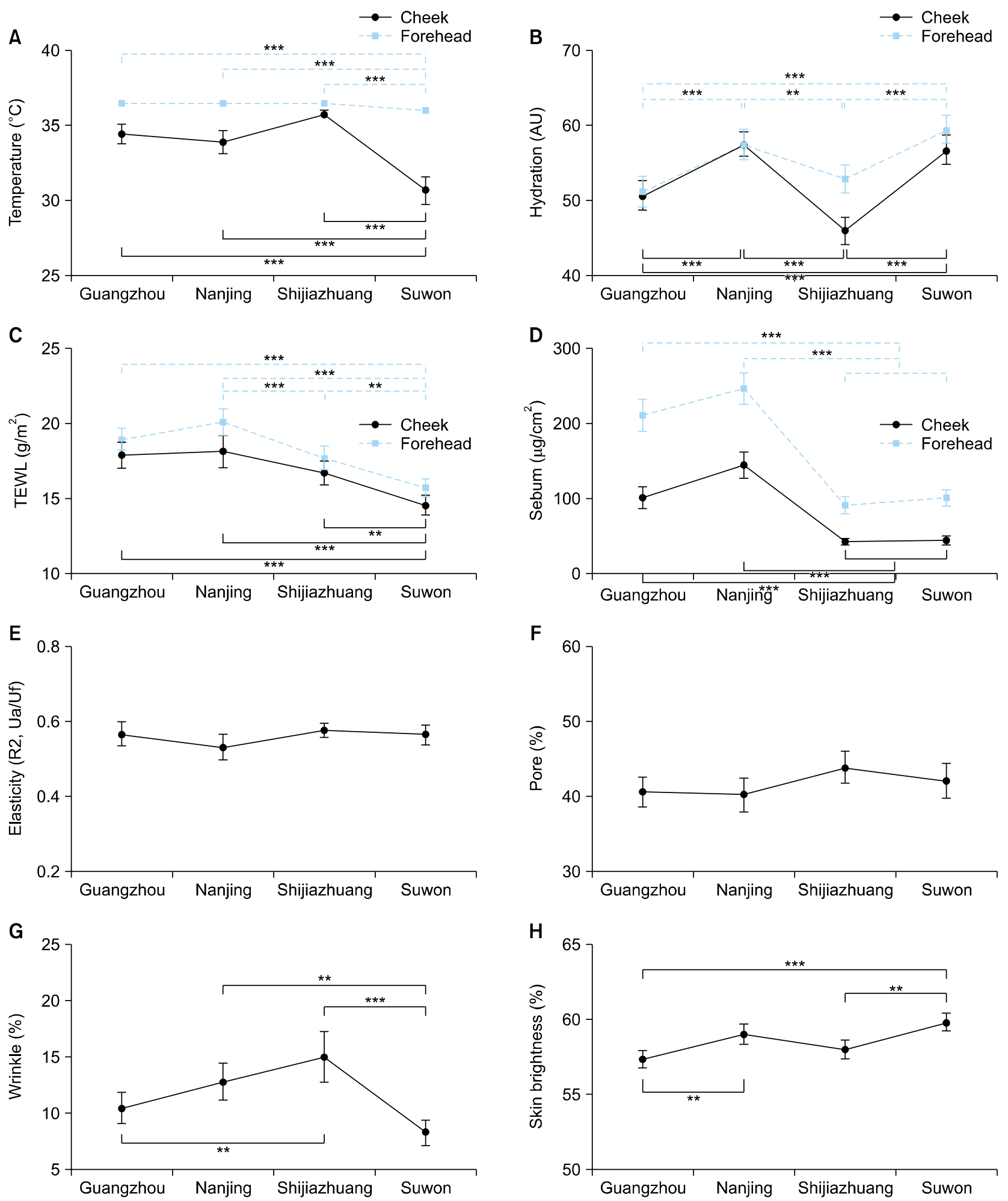

Fig. 3. Variation of biophysical parameters according to geographical region. (A, C) Skin surface temperature of the cheek and transepidermal water loss (TEWL) were lowest in Suwon compared to the 3 cities in China. (B) Hydration level was higher in warm cities (Guangzhou and Nanjing) than cold cities (Shijiazhuang and Suwon); in each city group, Nanjing and Suwon, cities which are more humid, demonstrated much higher hydration level. (D) Sebum excretion was higher in warm cities than cold cities. (E, F) Skin elasticity and pore size were not significantly different among the 4 cities. $(G, H)$ The severity of wrinkle was the mildest and skin tone brightest in Suwon compared to the 3 cities in China. ${ }^{* *} p<0.01,{ }^{* * *} p<0.001$. Error bar indicates a $95 \%$ confidence interval. 
Table 2. Age and biophysical parameters according to geographical region

\begin{tabular}{|c|c|c|c|c|c|}
\hline Parameters & Guangzhou $(n=92)$ & Nanjing $(\mathrm{n}=86)$ & Shijiazhuang $(n=95)$ & Suwon $(\mathrm{n}=88)$ & $p$-value \\
\hline Age $(y r)$ & $28.9 \pm 9.1$ & $29.1 \pm 9.5$ & $28.4 \pm 9.4$ & $30.4 \pm 9.5$ & 0.516 \\
\hline \multicolumn{6}{|c|}{ Temperature $\left({ }^{\circ} \mathrm{C}\right)$} \\
\hline Cheek & $34.5 \pm 3.2$ & $33.9 \pm 3.8$ & $35.9 \pm 0.6$ & $30.7 \pm 4.1$ & $0.000 * * *$ \\
\hline Forehead & $36.5 \pm 0.3$ & $36.5 \pm 0.3$ & $36.5 \pm 0.2$ & $36.1 \pm 0.6$ & $0.000 * * *$ \\
\hline \multicolumn{6}{|l|}{ Hydration (AU) } \\
\hline Cheek & $50.7 \pm 9.6$ & $57.5 \pm 7.9$ & $46.0 \pm 8.3$ & $56.8 \pm 9.1$ & $0.000 * * *$ \\
\hline Forehead & $51.2 \pm 10.1$ & $57.6 \pm 9.5$ & $52.9 \pm 9.0$ & $59.5 \pm 8.2$ & $0.000 * * *$ \\
\hline \multicolumn{6}{|l|}{ TEWL $\left(\mathrm{g} / \mathrm{m}^{2}\right)$} \\
\hline Cheek & $17.9 \pm 4.0$ & $18.1 \pm 5.0$ & $16.7 \pm 3.9$ & $14.6 \pm 3.1$ & $0.000 * * *$ \\
\hline Forehead & $18.9 \pm 3.4$ & $20.1 \pm 4.0$ & $17.7 \pm 3.9$ & $15.7 \pm 2.7$ & $0.000 * * *$ \\
\hline \multicolumn{6}{|c|}{ Sebum $\left(\mu \mathrm{g} / \mathrm{cm}^{2}\right)$} \\
\hline Cheek & $102.1 \pm 68.4$ & $145.5 \pm 79.1$ & $43.4 \pm 23.1$ & $44.7 \pm 26.9$ & $0.000 * * *$ \\
\hline Forehead & $210.9 \pm 104.0$ & $246.5 \pm 95.7$ & $92.3 \pm 54.0$ & $101.7 \pm 53.5$ & $0.000 * * *$ \\
\hline Elasticity (R2) & $0.6 \pm 0.1$ & $0.5 \pm 0.2$ & $0.6 \pm 0.1$ & $0.6 \pm 0.1$ & 0.140 \\
\hline Pore size $(\%)$ & $40.6 \pm 9.1$ & $40.1 \pm 10.4$ & $43.8 \pm 10.2$ & $42.0 \pm 10.6$ & 0.060 \\
\hline Wrinkle (\%) & $10.5 \pm 6.5$ & $12.6 \pm 7.6$ & $15.0 \pm 11.1$ & $8.3 \pm 5.1$ & $0.000 * * *$ \\
\hline Skin tone $(\%)$ & $57.3 \pm 2.9$ & $59.0 \pm 3.1$ & $57.9 \pm 3.1$ & $59.7 \pm 2.8$ & $0.000 * * *$ \\
\hline
\end{tabular}

Values are presented as mean \pm standard deviation. AU: arbitrary units, TEWL: transepidermal water loss. ${ }^{* * *} p<0.001$.

between Chinese (Guangzhou, Nanjing and Shijiazhuang) and Korean (Suwon) women (Fig. 4, Table 3). Korean women significantly used sunscreen more frequently than Chinese women (Fig. 4A). Furthermore, Korean women used sunscreen with higher sun protection factor (SPF) and protection grade of ultraviolet $A$ (PA) index compared to Chinese women (Fig. 4B, C). A majority of subjects were wearing base makeup daily, with a higher percentage in Korean women (base makeup means wearing a skin cover material, such as foundation, blemish balm cream, or compact) (Fig. 4D). The type of moisturizer was classified into 3 categories: 1 ) no use or toner only; 2) light moisturizers, lotion only or a combined use of toner and lotion; 3 ) heavy moisturizers, cream only, or a combined use of toner and cream, lotion and cream, or toner, lotion, and cream. Korean women used heavier moisturizers than Chinese women (Fig. 4E).

\section{Influence of cosmetic habits on biophysical parameters}

By performing multiple regression, the association between cosmetic habits and biophysical parameters (values measured in cheek were chosen) was assessed after adjusting for age and geographical region (Supplementary Table 2). Marginal mean was calculated and is shown in Fig. 5. Either subjects who used sunscreen sometimes or everyday showed significantly lower sebum excretion than subjects who never used sunscreen $(p=0.028$ and $p=0.010$, respectively) (Fig. 5A). Skin elasticity was significantly higher in subjects who used sunscreen everyday than subjects who never used sunscreen $(p=0.037)$
Table 3. Cosmetic habits according to country

\begin{tabular}{|c|c|c|c|}
\hline $\begin{array}{l}\text { Cosmetic } \\
\text { habits }\end{array}$ & $\begin{array}{l}\text { Chinese (Guangzhou, } \\
\text { Nanjing, and Shi- } \\
\text { jiazhuang; } n=273 \text { ) }\end{array}$ & $\begin{array}{l}\text { Korean } \\
\text { (Suwon; } \\
n=88 \text { ) }\end{array}$ & $p$-value \\
\hline \multicolumn{3}{|c|}{ Frequency of sunscreen usage } & $0.001 * *$ \\
\hline Never & $12(4.4)$ & $1(1.1)$ & \\
\hline Sometimes & $160(58.6)$ & $36(40.9)$ & \\
\hline Always & $101(37.0)$ & $51(58.0)$ & \\
\hline \multicolumn{3}{|c|}{ SPF index of sunscreen of usage ${ }^{\dagger}$} & $0.000^{* * *}$ \\
\hline$\leq 15$ & $7(2.6)$ & $1(1.1)$ & \\
\hline $16 \sim 30$ & $110(40.3)$ & $11(12.5)$ & \\
\hline $31 \sim 49$ & $44(16.1)$ & $23(26.1)$ & \\
\hline$\geq 50$ & $41(15.0)$ & $42(47.7)$ & \\
\hline \multicolumn{3}{|c|}{ PA index of sunscreen of usage ${ }^{\ddagger}$} & $0.002 * *$ \\
\hline 0 & $2(0.7)$ & $0(0)$ & \\
\hline+ & $23(8.4)$ & $1(1.1)$ & \\
\hline++ & $81(29.7)$ & $28(31.8)$ & \\
\hline+++ & $66(24.2)$ & $37(42.0)$ & \\
\hline \multicolumn{3}{|c|}{ Daily base makeup } & $0.015^{*}$ \\
\hline No & $34(12.5)$ & $3(3.4)$ & \\
\hline Yes & $239(87.5)$ & $85(96.6)$ & \\
\hline \multicolumn{3}{|c|}{ Types of moisturizer of usage } & $0.001 * *$ \\
\hline $\begin{array}{l}\text { No or toner } \\
\text { only }\end{array}$ & $15(5.5)$ & $1(1.1)$ & \\
\hline Light & $65(23.8)$ & $9(10.2)$ & \\
\hline Heavy & $193(70.7)$ & 78 (88.6) & \\
\hline
\end{tabular}

Values are presented as presented as number (\%). SPF: sun protection factor, PA: protection grade of ultraviolet $A .{ }^{*} p<0.05$, ${ }^{* *} p<0.01,{ }^{* * *} p<0.001 .{ }^{\dagger}$ Data available in 202 Chinese and 77 Korean patients. ${ }^{\ddagger}$ Data available in 172 Chinese and 66 Korean patients. 
A

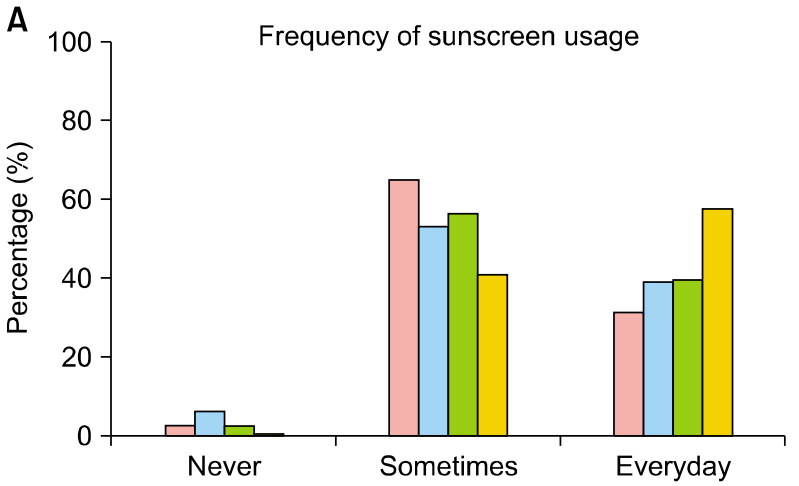

C
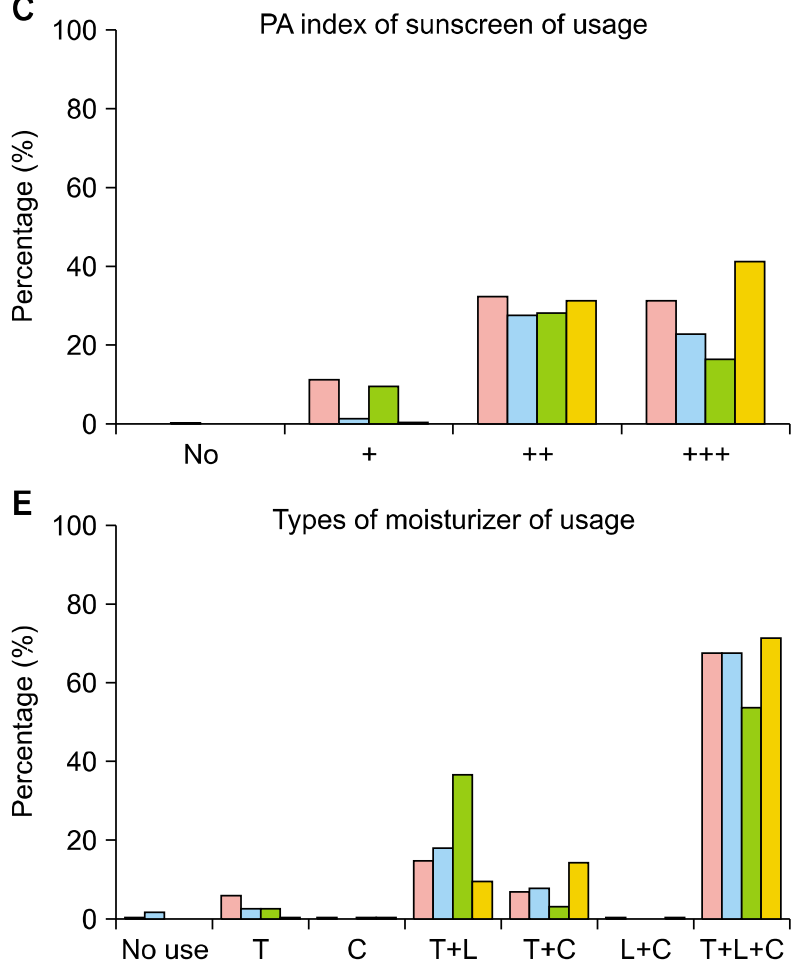

$B_{100} \quad$ SPF index of sunscreen of usage

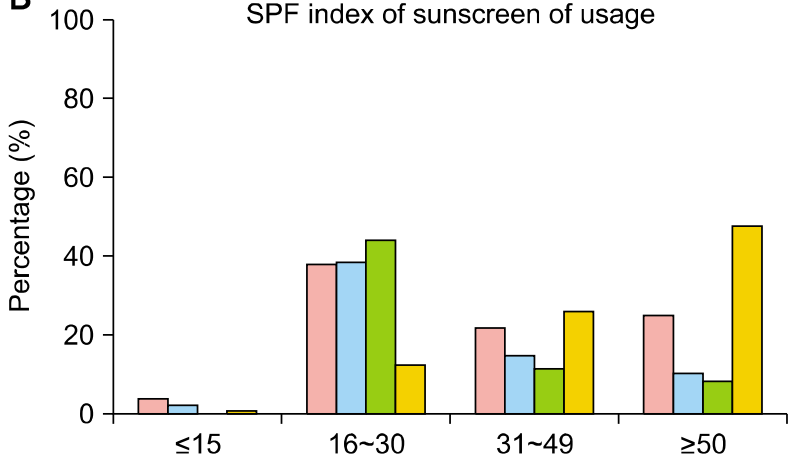

D $100 \quad$ Daily base makeup

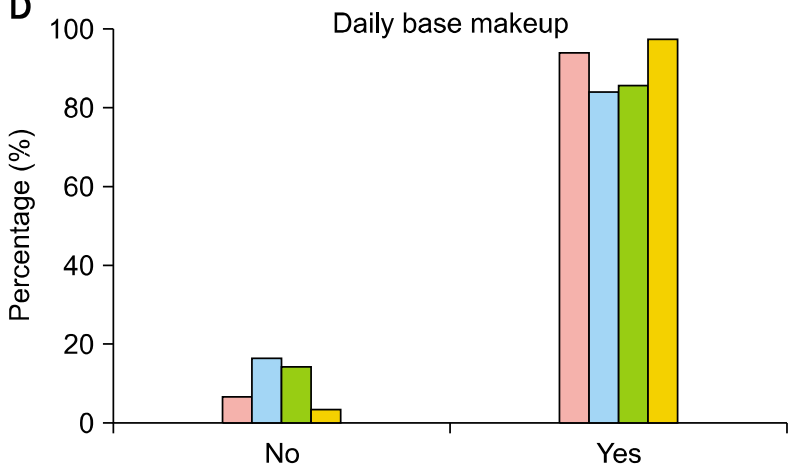

Fig. 4. Cosmetic habits according to geographical region. (A $\sim$ C) Subjects in Suwon had the greatest tendency to use sunscreen everyday and to use sunscreen with high SPF and PA index. (D) A majority of the subjects were shown to wear base makeup daily, with the highest percentage in Suwon. (E) Subjects in Suwon tended to use heavier moisturizers than the 3 cities of China, such as a combined use of toner and cream, or toner, lotion, and cream. T: toner, C: cream, L: lotion. SPF: sun protection factor, PA: protection grade of ultraviolet $\mathrm{A}$.

(Fig. 5A). Subjects who wore base makeup daily showed higher hydration level and lower TEWL than subjects who did not ( $p=0.004$ and $p=0.005$, respectively) (Fig. 5B). Either the subjects who used light moisturizers or those who used heavy moisturizers showed more hydration and lower TEWL than subjects who never used moisturizers or used toner only $(p=0.001$ and $p=0.036$ for hydration and TEWL, respectively, between light moisturizer group and no use or toner only group; and $p=0.001$ and $p=0.001$ for hydration and TEWL, respectively, between heavy moisturizer group and no use or toner only group) (Fig. 5C). However, the difference of hydration and TEWL between light and heavy moisturizers was not statistically significant ( $p=0.693$ and $p=0.05$, respectively). Skin elasticity was significantly higher in subjects who used heavy moisturizers than either the subjects who never used moisturizers or used toner only, or those who used light moisturizers ( $p=0.002$ and $p=0.036$, respectively) (Fig. 5D). Other biophysical parameters had no statistically significant correlation with cosmetic habits.

\section{DISCUSSION}

Several Asian studies have investigated association be- 

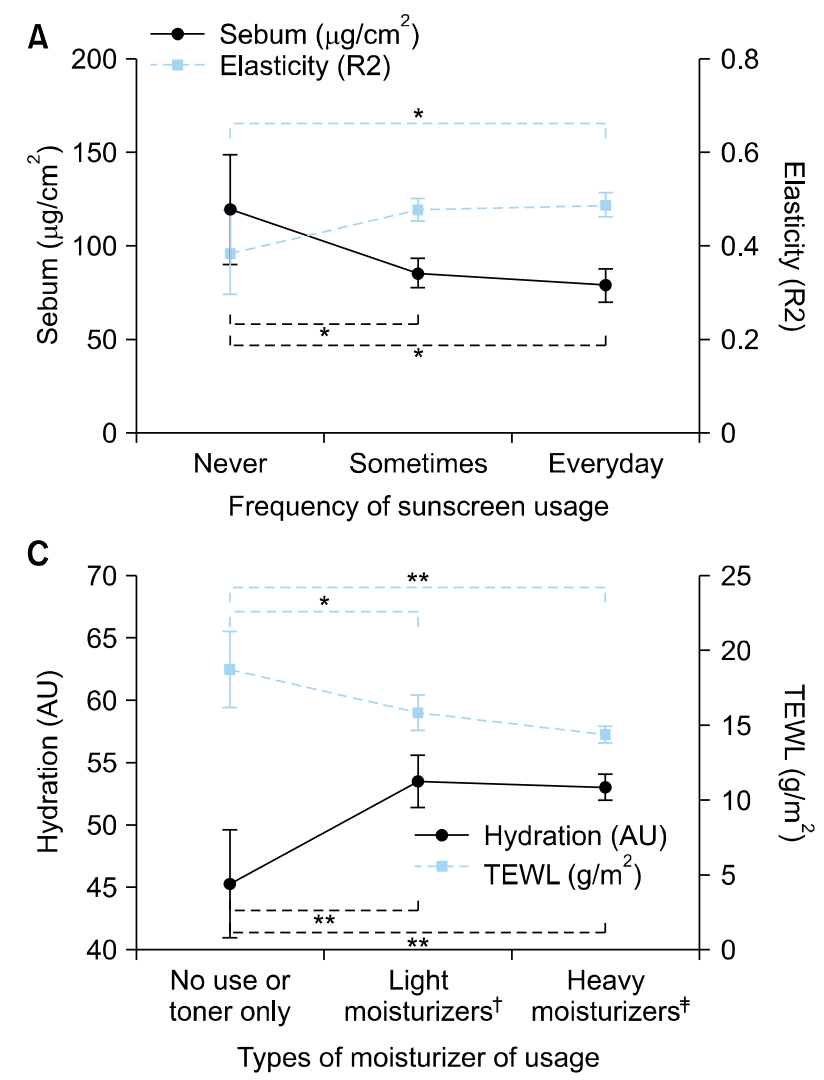
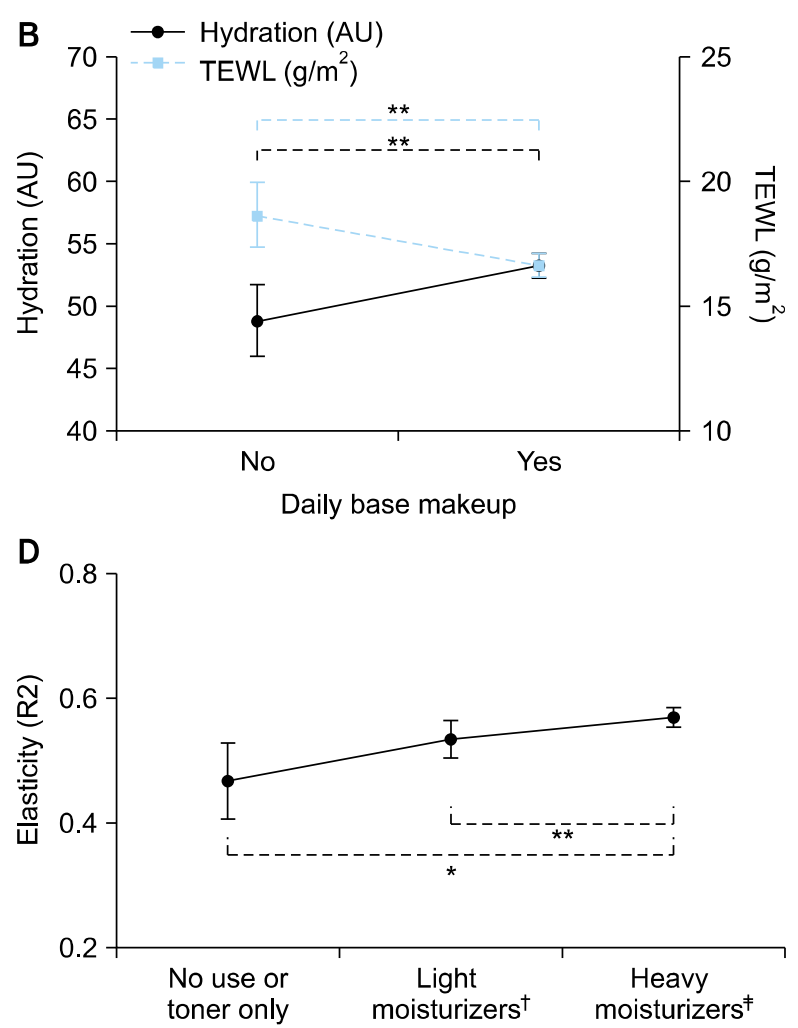

Types of moisturizer of usage

Fig. 5. Variations of biophysical parameters according to cosmetic habit. (A) Use of sunscreen either sometimes or everyday was associated with low sebum excretion. Use of sunscreen everyday was related to high elasticity. (B) Wearing base makeup daily was linked to high hydration level and low transepidermal water loss (TEWL). (C) Either use of light or heavy moisturizers was associated with high hydration level and low TEWL, with no significant difference between the two types of moisturizers. (D) Use of heavy moisturizers was related to high elasticity. ${ }^{*} p<0.05,{ }^{*} p<0.01,{ }^{\dagger}$ Include use of lotion only, and a combined use of toner and lotion, ${ }^{\ddagger}$ Include use of cream only, and a combined use of toner and cream, lotion and cream, and toner, lotion, and cream. Error bar indicates a $95 \%$ confidence interval.

tween cutaneous biophysical parameters and age, climate, or skin care habits, but most studies have included only small number of participants who are from a one nation ${ }^{2,6,16}$. Our study is the first study that investigated biophysical parameters in a large number of Korean and Chinese women (northeast Asian).

Although both Suwon and Shijiazhuang showed similar low mean temperature, skin surface temperature, especially the values measured in the cheek, was significantly lower in Suwon. Given that wind can contribute to surface temperature lowering when ambient temperature is $l^{2} w^{21}$, the wind of Suwon which is twice stronger than Shijiazhuang could have lowered the skin surface temperature more than in Shijiazhuang. However, forehead cooled less than cheek owing to high vascularity and thin subcutaneous fat layer ${ }^{21,22}$. Abundant fat tissues in the cheek could have limited heat transfer from the deep tissues to the $\operatorname{skin}^{21,22}$.
Hydration of the stratum corneum is important for maintenance of the skin barrier ${ }^{6,23,24}$. In the present study, hydration level showed no significant difference according to age. The results of previous studies are controversial: some authors found no relation between skin hydration and age $^{7-9}$; in contrast some others found hydration variation with age $\mathrm{e}^{5,6,10}$. Most of the latter revealed that hydration level rapidly decreased in the ages of $60 \sim 80$ s, which was not a continuous decrease with age. Overall, given the relatively young age (less than 50 years) of subjects in this study, hydration level does not appear to be influenced by aging, at least in the young ages. According to a previous study, hydration level has a positive correlation with temperature and relative humidity ${ }^{16}$. That can be an appropriate explanation for our results: hydration level was higher in humid cities.

TEWL is a well-known in vivo indicator of skin barrier function $^{25,26}$. Although there have been a few controver- 
sies regarding the association of TEWL and aging, many authors found no significant relation between them, which is consistent with our results ${ }^{5,10}$. Furthermore, TEWL has been known to have negative correlation with temperature and relative humidity ${ }^{16}$. However, in the present study, Suwon showed conspicuously lower TEWL than the 3 cities in China. This implies that habits or ethnicity could have an impact besides climate. Subjects in Suwon had a tendency to use heavy moisturizers and showed the highest percentage of women wearing base makeup daily. Some previous studies has reported that skin sebum excretion is reduced after menopause in women ${ }^{6,11,12}$, which could be linked to hormonal influence. However, almost all of the subjects in this study were in pre-menopause state, and there was no significant difference in sebum excretion according to age group. In comparison between cities, warm cities showed higher sebum content ${ }^{27}$. However, the difference of sebum excretion due to humidity difference in each city group was small, which differs from the results of the previous study ${ }^{16}$. Women who used sunscreen showed lower sebum output than women who never used one. This could be more likely due to the low usage of sunscreen by those who have oily skin, rather than a direct sebum-reducing effect of sunscreen.

Although the skin elasticity and tone decreased and pore and wrinkle increased with age, wrinkle showed the most distinct change according to age. Wrinkle seems to be the most representative parameter which reflects skin aging. There is a possible explanation that variations of biophysical parameters including elasticity, pore size, and skin tone with aging are ultimately linked to a change in skin appearance, i.e., wrinkle.

In comparison of the 4 cities, Women in Suwon showed a better profile of biophysical parameters than women in China. Women in Suwon showed higher levels of hydration and lower levels of TEWL and sebum. Also, wrinkle and skin tone values were notably better in the subjects in Suwon than those in the 3 cities of China. In terms of habits, women in Suwon had a tendency to use heavy moisturizers, to use sunscreen everyday, and to use sunscreen with high SPF and PA indices. Given the results of the current study demonstrating that cosmetic habits such as using sunscreen and moisturizers, and wearing base makeup daily improved several biophysical parameters regardless of climates or geographic regions ${ }^{2,28,29}$, habits of using cosmetics in women in Suwon might have contributed to the better profiles of biophysical parameters ${ }^{15,30,31}$.

There have been various studies investigating various factors that could influence the biophysical parameters other than age, climatic factors, or cosmetic habits: body region $^{5,10,13,30}$, seasonal and daytime variation ${ }^{1,16}$, daily out- side activities ${ }^{2}$, water consumption ${ }^{2}$, sensitive skin $^{31}$, di$\mathrm{et}^{31}$, or detergents ${ }^{32}$. Also, some details of cosmetic habits which were not collected in this study, such as the amount or frequency of application, could influence biophysical parameters. Moreover, socioeconomic status, educational level, or occupation could additionally affect the parameters.

The study is a cross-sectional study and therefore cannot imply causation. With respect to some cosmetic habits, the number of subjects who had a particular sub-item of habits (e.g., subjects who never used sunscreen, who used no moisturizers or used toner only, who used SPF $\leq 15$ sunscreen, or who used PA 0 sunscreen) was relatively small that bias could interfere in group comparison. However, the results can be understood all together as an overall increasing or decreasing tendency of biophysical parameters according to the changes in other sub-items of habits where subjects are sufficient in number. Moreover, the present study has several strengths: (1) a relatively large number of northeast Asian women were enrolled; (2) objective biophysical parameters were compared between Chinese and Korean women, which demonstrated that cosmetic habits were significantly associated with the differences in these parameters.

In conclusion, we demonstrated that Korean women (Suwon) have a higher hydration level, lower TEWL and sebum, less severe wrinkle and brighter skin tone than the 3 cities in China (Guangzhou, Nanjing, and Shijiazhuang). These could be attributed to cosmetic habits, besides age and climatic factors. An appropriate use of cosmetics, such as using sunscreen with high SPF and PA index everyday and using moisturizers, could help to improve skin functions.

\section{ACKNOWLEDGMENT}

This study was funded by SKIN79, Inc.

\section{SUPPLEMENTARY MATERIALS}

Supplementary data can be found via http://anndermatol. org/src/sm/ad-31-175-s001.pdf.

\section{CONFLICTS OF INTEREST}

The authors have nothing to disclose.

\section{ORCID}

Ji Su Lee, https://orcid.org/0000-0003-0207-2107

Jaehyoun Ha, https://orcid.org/0000-0003-3019-8217 
Kyeho Shin, https://orcid.org/0000-0001-5082-0769

Hyojung Kim, https://orcid.org/0000-0001-5645-5299

Soyun Cho, https://orcid.org/0000-0003-2468-485X

\section{REFERENCES}

1. Firooz A, Zartab H, Sadr B, Bagherpour LN, Masoudi A, Fanian $F$, et al. Daytime changes of skin biophysical characteristics: a study of hydration, transepidermal water loss, $\mathrm{pH}$, sebum, elasticity, erythema, and color index on middle eastern skin. Indian J Dermatol 2016;61:700.

2. Hadi H, Awadh Al, Hanif NM, Md Sidik NF, Mohd Rani MR, Suhaimi MS. The investigation of the skin biophysical measurements focusing on daily activities, skin care habits, and gender differences. Skin Res Technol 2016;22:247-254.

3. Boer M, Duchnik E, Maleszka R, Marchlewicz M. Structural and biophysical characteristics of human skin in maintaining proper epidermal barrier function. Postepy Dermatol Alergol 2016;33:1-5.

4. Baek JH, Lee MY, Koh JS. Relationship between clinical features of facial dry skin and biophysical parameters in Asians. Int J Cosmet Sci 2011;33:222-227.

5. Firooz A, Sadr B, Babakoohi S, Sarraf-Yazdy M, Fanian F, Kazerouni-Timsar A, et al. Variation of biophysical parameters of the skin with age, gender, and body region. ScientificWorldJournal 2012;2012:386936.

6. Man MQ, Xin SJ, Song SP, Cho SY, Zhang XJ, Tu CX, et al. Variation of skin surface $\mathrm{pH}$, sebum content and stratum corneum hydration with age and gender in a large Chinese population. Skin Pharmacol Physiol 2009;22:190-199.

7. Wilhelm KP, Cua AB, Maibach HI. Skin aging. Effect on transepidermal water loss, stratum corneum hydration, skin surface $\mathrm{pH}$, and casual sebum content. Arch Dermatol 1991;127:1806-1809.

8. Wendling PA, Dell'Acqua G. Skin biophysical properties of a population living in Valais, Switzerland. Skin Res Technol 2003;9:331-338.

9. Mayes AE, Murray PG, Gunn DA, Tomlin CC, Catt SD, Wen $\mathrm{YB}$, et al. Ageing appearance in China: biophysical profile of facial skin and its relationship to perceived age. J Eur Acad Dermatol Venereol 2010;24:341-348.

10. Marrakchi S, Maibach HI. Biophysical parameters of skin: map of human face, regional, and age-related differences. Contact Dermatitis 2007; 57:28-34.

11. Ohta H, Makita K, Kawashima T, Kinoshita S, Takenouchi M, Nozawa S. Relationship between dermato-physiological changes and hormonal status in pre-, peri-, and postmenopausal women. Maturitas 1998;30:55-62.

12. Zouboulis CC, Boschnakow A. Chronological ageing and photoageing of the human sebaceous gland. Clin Exp Dermatol 2001;26:600-607.

13. Lee MR, Nam GW, Jung YC, Park SY, Han JY, Cho JC, et al. Comparison of the skin biophysical parameters of Southeast Asia females: forehead-cheek and ethnic groups. J Eur Acad Dermatol Venereol 2013;27:1521-1526.

14. Nouveau-Richard S, Yang Z, Mac-Mary S, Li L, Bastien P,
Tardy I, et al. Skin ageing: a comparison between Chinese and European populations. A pilot study. J Dermatol Sci 2005; 40:187-193.

15. Akazaki $S$, Nakagawa $H$, Kazama $H$, Osanai $O$, Kawai $M$, Takema $\mathrm{Y}$, et al. Age-related changes in skin wrinkles assessed by a novel three-dimensional morphometric analysis. Br J Dermatol 2002;147:689-695.

16. Wan MJ, Su XY, Zheng Y, Gong ZJ, Yi JL, Zhao Y, et al. Seasonal variability in the biophysical properties of forehead skin in women in Guangzhou City, China. Int J Dermatol 2015;54:1319-1324.

17. Sun JY, Kim SW, Lee SH, Choi JE, Ko SJ. Automatic facial pore analysis system using multi-scale pore detection. Skin Res Technol 2017;23:354-362.

18. Bae SH, Park JJ, Song EJ, Lee JA, Byun KS, Kim NS, et al. The comparison of the melanin content and UV exposure affecting aging process: seven countries in Asia. J Cosmet Dermatol 2016;15:335-342.

19. Song EJ, Lee JA, Park JJ, Kim HJ, Kim NS, Byun KS, et al. A study on seasonal variation of skin parameters in Korean males. Int J Cosmet Sci 2015;37:92-97.

20. Kim HK, Choi JH. Effects of radiofrequency, electroacupuncture, and low-level laser therapy on the wrinkles and moisture content of the forehead, eyes, and cheek. J Phys Ther Sci 2017;29:290-294.

21. Brajkovic D, Ducharme MB. Facial cold-induced vasodilation and skin temperature during exposure to cold wind. Eur J Appl Physiol 2006;96:711-721.

22. LeBlanc J, Blais B, Barabé B, Côté J. Effects of temperature and wind on facial temperature, heart rate, and sensation. J Appl Physiol 1976;40:127-131.

23. Darlenski R, Fluhr JW. Influence of skin type, race, sex, and anatomic location on epidermal barrier function. Clin Dermatol 2012;30:269-273.

24. Parente ME, Gambaro A, Ares G. Sensory characterization of emollients. J Sens Stud 2008;23:149-161.

25. Pinnagoda J, Tupker RA, Agner T, Serup J. Guidelines for transepidermal water loss (TEWL) measurement. A report from the Standardization Group of the European Society of Contact Dermatitis. Contact Dermatitis 1990;22:164-178.

26. Fluhr JW, Feingold KR, Elias PM. Transepidermal water loss reflects permeability barrier status: validation in human and rodent in vivo and ex vivo models. Exp Dermatol 2006;15: 483-492.

27. Cunliffe WJ, Burton JL, Shuster S. The effect of local temperature variations on the sebum excretion rate. $\mathrm{Br}$ J Dermatol 1970;83:650-654.

28. Jemec GB, $\mathrm{Na}$ R. Hydration and plasticity following long-term use of a moisturizer: a single-blind study. Acta Derm Venereol 2002;82:322-324.

29. Rosado C, Pinto P, Rodrigues LM. Assessment of moisturizers and barrier function restoration using dynamic methods. Skin Res Technol 2009;15:77-83.

30. Kleesz P, Darlenski R, Fluhr JW. Full-body skin mapping for six biophysical parameters: baseline values at 16 anatomical sites in 125 human subjects. Skin Pharmacol Physiol 2012;25:25-33. 
31. Nouveau-Richard S, Zhu W, Li YH, Zhang YZ, Yang FZ, Yang ZL, et al. Oily skin: specific features in Chinese women. Skin Res Technol 2007;13:43-48.
32. Löffler $H$, Dickel $H$, Kuss $O$, Diepgen TL, Effendy I. Characteristics of self-estimated enhanced skin susceptibility. Acta Derm Venereol 2001;81:343-346. 
Supplementary Table 1. Simple linear regression of age for biophysical parameters

\begin{tabular}{|c|c|c|c|c|}
\hline Dependent variable & R-squared & B & $\mathrm{t}$ & $p$-value \\
\hline \multicolumn{5}{|l|}{ Temperature $\left({ }^{\circ} \mathrm{C}\right)$} \\
\hline Cheek & 0.032 & 35.891 & 56.795 & $0.000 * * *$ \\
\hline Forehead & 0.019 & -0.006 & -2.633 & $0.009 * *$ \\
\hline \multicolumn{5}{|l|}{ Hydration (AU) } \\
\hline Cheek & 0.006 & 0.080 & 1.425 & 0.155 \\
\hline Forehead & 0.006 & 0.082 & 1.497 & 0.135 \\
\hline \multicolumn{5}{|l|}{ TEWL $\left(\mathrm{g} / \mathrm{m}^{2}\right)$} \\
\hline Cheek & 0.003 & -0.025 & -1.053 & 0.293 \\
\hline Forehead & 0.019 & -0.056 & -2.610 & $0.009 * *$ \\
\hline \multicolumn{5}{|l|}{ Sebum $\left(\mu \mathrm{g} / \mathrm{cm}^{2}\right)$} \\
\hline Cheek & 0.026 & -1.196 & -3.091 & $0.002 * *$ \\
\hline Forehead & 0.043 & -2.306 & -3.994 & $0.000 * * *$ \\
\hline Elasticity (R2) & 0.126 & -0.005 & -7.190 & $0.000 * * *$ \\
\hline Pore size (\%) & 0.297 & 0.591 & 12.330 & $0.000 * * *$ \\
\hline Wrinkle (\%) & 0.455 & 0.600 & 17.314 & $0.000 * * *$ \\
\hline Skin tone $(\%)$ & 0.141 & -0.124 & -7.663 & $0.000 * * *$ \\
\hline
\end{tabular}

R-squared: determination coefficient, B: unstandardized regression coefficient, $\mathrm{t}=\mathrm{B} /$ standard error, $\mathrm{AU}$ : arbitrary units, TEWL: transepidermal water loss. ${ }^{* *} p<0.01,{ }^{* * *} p<0.001$. 
JS Lee, et al

Supplementary Table 2. Association between cosmetic habits and biophysical parameters after adjusting for age and geographical region

\begin{tabular}{|c|c|c|c|c|c|c|c|c|}
\hline & \multicolumn{2}{|c|}{ Hydration (AU) } & \multicolumn{2}{|c|}{ TEWL $\left(\mathrm{g} / \mathrm{m}^{2}\right)$} & \multicolumn{2}{|c|}{ Sebum $\left(\mu \mathrm{g} / \mathrm{cm}^{2}\right)$} & \multicolumn{2}{|c|}{ Elasticity (R2, ratio) } \\
\hline & Beta & $p$-value & Beta & $p$-value & Beta & $p$-value & Beta & $p$-value \\
\hline \multicolumn{9}{|c|}{ Frequency of sunscreen usage } \\
\hline Never & (Reference) & (Reference) & (Reference) & (Reference) & (Reference) & (Reference) & (Reference) & (Reference) \\
\hline Sometimes & -0.104 & 0.401 & -0.149 & 0.276 & -0.246 & $0.028^{*}$ & 0.254 & 0.058 \\
\hline Always & 0.107 & 0.389 & -0.208 & 0.129 & -0.289 & $0.010^{*}$ & 0.281 & $0.037^{*}$ \\
\hline \multicolumn{9}{|l|}{ Daily base makeup } \\
\hline No & (Reference) & (Reference) & (Reference) & (Reference) & (Reference) & (Reference) & (Reference) & (Reference) \\
\hline Yes & 0.134 & $0.004 * *$ & -0.142 & $0.005^{* *}$ & 0.025 & 0.589 & 0.054 & 0.284 \\
\hline \multicolumn{9}{|c|}{ Types of moisturizer of usage } \\
\hline No or toner only & (Reference) & (Reference) & (Reference) & (Reference) & (Reference) & (Reference) & (Reference) & (Reference) \\
\hline Light & 0.333 & $0.001^{* *}$ & -0.222 & $0.036^{*}$ & -0.135 & 0.124 & 0.199 & 0.055 \\
\hline Heavy & 0.336 & $0.001 * *$ & -0.348 & $0.001 * *$ & -0.158 & 0.072 & 0.329 & $0.002 * *$ \\
\hline
\end{tabular}

AU: arbitrary units, TEWL: transepidermal water loss, Beta: standardized regression coefficient. ${ }^{*} p<0.05,{ }^{*} p<0.01$. 